\title{
Calculation of stress in FGM beams
}

\author{
Justín Murín ${ }^{1, *}$, Juraj Hrabovský ${ }^{1}$, Vladimir Kutiš ${ }^{1}$ \\ ${ }^{1}$ Department of Applied Mechanics and Mechatronics, Institute of Automotive Mechatronics, Faculty \\ of Electrical Engineering and Information Technology, Slovak University of Technology \\ in Bratislava, Ilkovičova 3, 81219 Bratislava, Slovakia
}

\begin{abstract}
Content of the paper is oriented to calculation of elastic normal stress in the Functionally Graded Beams (FGM). Spatial variation of material properties is considered in the lateral, transversal and longitudinal direction of the straight beam. The displacements and internal forces are calculated using our new FGM finite beam element. Heterogeneous material properties are homogenized by extended mixture rules, laminate theory and reference volume element (RVE). Obtained results by our approach are evaluated and compared with the ones obtained by the 3D solid finite elements.
\end{abstract}

Keywords: FGM beams, Spatially varying material properties, Normal and shear stress calculation

\section{Introduction}

Many papers deal with static analysis of the FGM single 2D beams with transverse variation of material properties only [1-5]. Less attention is paid to longitudinal and lateral variation of material properties. However, the authors did not find papers which consequently deal with all the longitudinal, transversal and lateral variation of material properties by single beams or beam structures built of such FGM.

The presented contribution is the continuation of our previous work dealing with the derivation of a 3D FGM beam finite element with longitudinal varying effective material properties, which is suitable for analysis of beam structures made of spatially varying FGM. Effect of the axial and shear forces and Winkler elastic foundation is included, as well. Homogenization of the spatial varying material properties in the real FGM beam and the calculation of effective parameters are done by the multilayer or direct integration method (MLM or DIM) [6]. If only transversal and lateral variations of material properties are considered in the real FGM beam, longitudinally constant effective material properties arise from the homogenization. This method can also be used in the homogenization of multilayer beams with discontinuous variation of material properties in transversal and lateral direction. Numerical experiments are performed to calculate the elastostatic response of chosen spatial FGM beam structures with rectangular cross-sections with symmetrically lateral, transversal and longitudinal variations of material properties. Expressions for normal stress calculation in the cross-section caused by the axial force as well by the

\footnotetext{
* Corresponding author: justin.murin@stuba.sk

Reviewers: Robert Zalewski, Milan Žmindák
} 
bending moments are established. For torsional loading only the twist angle and internal torsional moment is evaluated. The solution results are discussed and compared to those obtained by means of very fine $3 \mathrm{D}$ - solid finite element mesh.

\section{3D FGM beam finite element with spatially varying material properties}

Let us consider a 3D straight finite beam element (Timoshenko beam theory and SaintVenant torsion theory) of doubly symmetric cross-section - Fig. 1. The nodal degrees of freedom at node $i$ are: the displacements $u_{i}, v_{i}, w_{i}$ in the local axis direction $x, y, z$ and the cross-sectional area rotations $-\varphi_{x, i}, \varphi_{y, i}, \varphi_{z, i}$. The degrees of freedom at the node $j$ are denoted in a similar manner. The internal forces at node $i$ are: the axial force $N_{i}$, the transversal forces $R_{y, i}$ and $R_{z, i}$, the bending moments $M_{y, i}$ and $M_{z, i}$, and the torsion moment $M_{x, i}$. The first derivative with respect to $x$ of the relevant variable is denoted with an apostrophe "'"'.

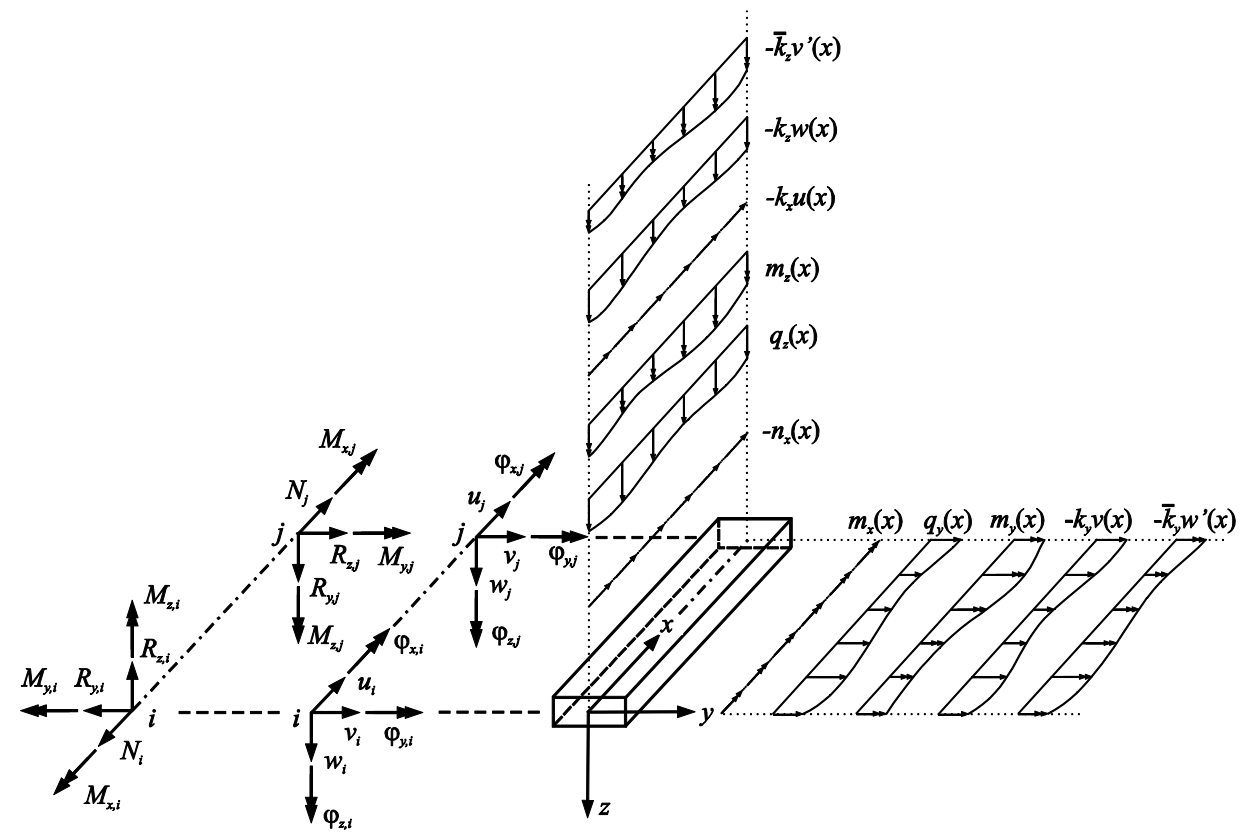

Fig. 1. The local internal variables and loads

Furthermore, $n_{x}=n_{x}(x)$ is the axial force distribution, $q_{z}=q_{z}(x)$ and $q_{y}=q_{y}(x)$ are the transversal and lateral force distributions, $m_{x}=m_{x}(x), m_{y}=m_{y}(x)$ and $m_{z}=m_{z}(x)$ are the distributed moments, $A$ is the cross-sectional area, $I_{y}$ and $I_{z}$ are the second area moments, $I_{p}=I_{y}+I_{z}$ is the polar area moment, $k_{x}=k_{x}(x), k_{y}=k_{y}(x), k_{z}=k_{z}(x), \bar{k}_{y}=\bar{k}_{y}(x), \bar{k}_{z}=\bar{k}_{z}(x)$ are the elastic foundation modules (the torsional elastic foundation is not considered). The effective homogenized and longitudinally varying stiffness reads: $E A=E_{L}^{N H}(x) A$ is the axial stiffness $\left(E_{L}^{N H}(x) \equiv E_{L}^{N H}\right.$ is the effective elasticity modulus for axial loading), $E I_{y}=E_{L}^{M_{y} H}(x) I_{y}$ is the flexural stiffness about the $y$-axis $\left(E_{L}^{M_{y} H}(x) \equiv E_{L}^{M_{y} H}\right.$ is the effective elasticity modulus for bending 
about axis $y), E I_{z}=E_{L}^{M_{z} H}(x) I_{z}$ is the flexural stiffness about the axis $z,\left(E_{L}^{M_{z} H}(x) \equiv E_{L}^{M_{z} H}\right.$ is the effective elasticity modulus for bending about axis $z), G \bar{A}_{y}=G_{L y}^{H}(x) k_{y}^{s m} A$ is the reduced shear stiffness in $y$-direction $\left(G_{L y}^{H}(x) \equiv G_{L y}^{H}\right.$ is the effective shear modulus and $k_{y}^{s m}$ is the average shear correction factor in $y$-direction), $G \bar{A}_{z}=G_{I z}^{H}(x) k_{z}^{s m} A$ is the reduced shear stiffness in $z$-direction $\left(G_{L z}^{H}(x)=G_{L z}^{H}\right.$ is the effective shear modulus and $k_{y}^{s m}$ is the average shear correction factor in $z$-direction), $G_{L}^{M_{x} H}(x) I_{T}$ is the effective torsional stiffness ( $G_{L}^{M_{x} H}(x)=G_{L}^{M_{x} H}$ is the torsional elasticity modulus and $I_{T}$ is the torsion constant).

Mixture rules are one of the methods for micromechanical modeling of heterogeneous materials. Extended mixture rules [7] are based on the assumption that the constituents volume fractions (formally only denoted here as fibres - $\mathrm{f}$ and matrix $-\mathrm{m}$ ) continuously vary as polynomial functions, $v_{f}(x, y, z)$ and $v_{m}(x, y, z)$. The condition $v_{f}(x, y, z)+v_{m}(x, y, z)=1$ has to be fulfilled. The appropriated material property distribution in the real FGM beam (Fig. 2a.) then reads

$$
p(x, y, z)=v_{f}(x, y, z) p_{f}(x, y, z)+v_{m}(x, y, z) p_{m}(x, y, z)
$$

Here, $p_{f}(x, y, z)$ and $p_{m}(x, y, z)$ are the spatial distributions of material properties of the FGM constituents. The extended mixture rule (1) can be analogically used for FGM material made of more than two constituents. The assumption of a polynomial variation of the constituent's volume fractions and material properties enables an easier establishing of the main appropriated field equations and allows the modeling of many common realizable variations.

In literature and in practical applications, mostly the one directional variation of the FGM properties is considered. There, an exponential law for transversal variation of the constituents volume fractions is often presented, e.g. in $[8,9,10]$ and in references therein.

For the FGM beams the transversal variation (continuously or discontinuously, symmetrically or asymmetrically) has been mainly considered. The homogenization of such material properties variation is relatively simple. If the material properties vary only with respect to the longitudinal direction, the homogenization is frequently not needed since there are new FGM beam and link finite elements established that consider such variations in a very accurate and effective way, e.g. in $[6,11]$. A more complicated case is, if the material properties vary in three directions - namely in transversal, lateral and longitudinal direction of the FGM real beam and the torsion is included as well.

In this contribution, the homogenization techniques for spatially varying (continuously or discontinuously and symmetrically in transversal and lateral direction, and continuously in longitudinal direction) material properties of FGM beams of selected doubly-symmetric cross-sections are considered. The expressions are proposed for the derivation of effective elasticity modules for axial loading, the transversal and lateral bending, the shear modules for transversal and lateral shear and for uniform torsion by the extended mixture rules (EMR) and the multilayer method (MLM).

Let us consider a two nodded $3 \mathrm{D}$ straight beam element with double symmetric crosssectional area $A$ (Fig. 2). The composite material of this beam arises from mixing two components. The continuous polynomial spatial variation of the elasticity moduli and mass density can be caused by continuous polynomial spatial variation of both the volume fraction $\left(v_{f}(x, y, z)\right.$ and $\left.v_{m}(x, y, z)\right)$ and material properties of the FGM constituents ( $p_{f}(x, y, z)$ and $\left.p_{m}(x, y, z)\right)$. 
In our case the elasticity modulus $E(x, y, z)$, the Poisson ratio for the real beam have been calculated by expression (1). The FGM shear modulus can be calculated by expression:

$$
G(x, y, z)=\frac{E(x, y, z)}{2(1+v(x, y, z))} .
$$

If the constituents Poisson's ratio are approximately of the same value and the constituent volume fractions variation is not strong, then the FGM shear modulus can by calculated using a simplification [12]:

$$
G(x, y, z)=\frac{E(x, y, z)}{\xi},
$$

where $\xi$ is an average value of the function $\xi(x, y, z)=2(1+v(x, y, z))$

$$
\xi=\frac{1}{L} \int_{0}^{L}\left(\frac{1}{A} \int_{(A)} \xi(x, y, z) d A\right) d x .
$$

a) real beam

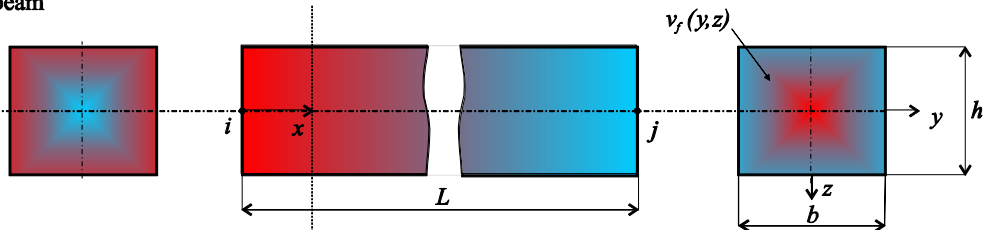

b) multilayer beam
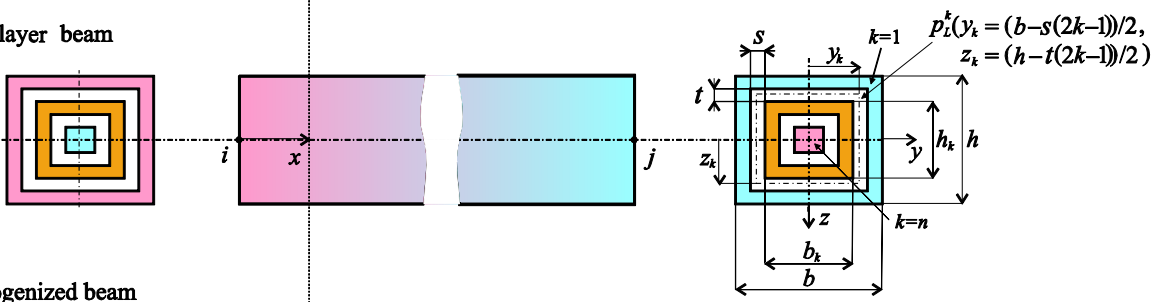

c) homogenized beam

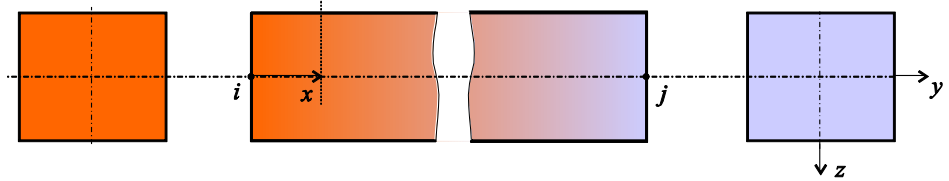

Fig. 2. FGM beam with rectangular cross-section

Homogenization of the spatially varying material properties (the reference volume is the volume of the whole beam) are done in two steps. In the first step, the real beam (Fig. 2a) is transformed into a multilayer beam (Fig. 2b). Material properties of the layers are calculated with the EMR [13]. We assume that each layer cross-section at position $x$ has constant material properties. They are calculated as an average value from their values at the boundaries of the respective layer cross-section. Polynomial variation of these properties appears in the longitudinal direction of the layer. Sufficient accuracy of the proposed substitution of the continuous transversal and lateral variation of material properties by the layer-wise constant distribution of material properties is reached if the division to layers is fine enough. In the second step, the effective longitudinal material properties of the homogenized beam are derived using the MLM [14]. These homogenized material properties are constant through the beam's height and depth but they vary continuously along the longitudinal beam axis. Accordingly, the beam finite element 
equation is established for the homogenized beam (Fig. 2c) in order to calculate the primary beam unknowns - the displacements and internal forces and critical buckling force in our case. The stress has to be calculated on the real beam and it is the main goal of this paper that will be presented in the next chapter.

\section{Normal stress calculation in the FGM rectangular cross- sectional area}

Stress calculation depends of the cross-sectional area form. In the next we focus on the calculation of normal stress distribution on rectangular cross section. Calculation of the shear and torsional stress in the FGM beams is more complicated in our approach. We deal with this task very active and the results will be published in our future publication.

The rectangular cross-section at position $x$ with doubly symmetric variation of material properties is considered - Fig. 3. At the centroid acts the normal force $N(x)$, the bending moments $M_{y}(x)$ and $M_{z}(x) . A=b h$ is the cross-sectional area, $I_{z}=b h^{3} / 12$ and $I_{y}=b^{3} h / 12$ are the quadratic moments of the rectangular cross-section.

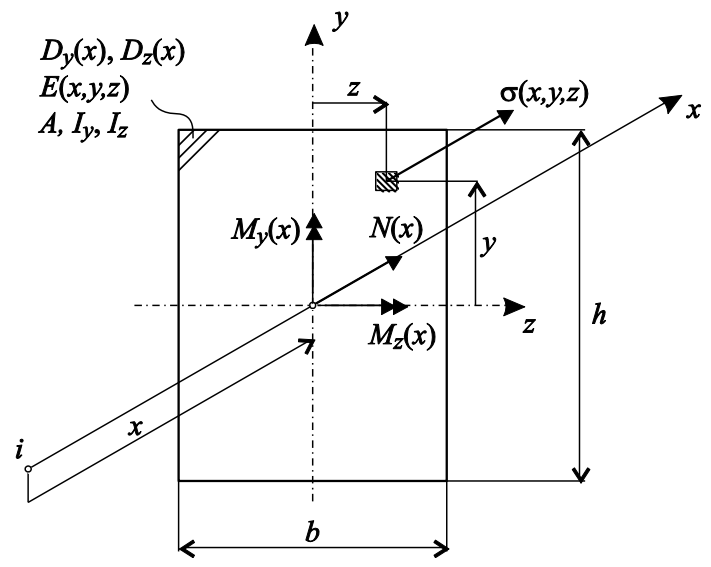

Fig. 3. To normal stress calculation

The resultant normal stress at point $(x, y, z)$ is the sum:

$$
\sigma(x, y, z)=\sigma^{N}(x, y, z)+\sigma^{M_{z}}(x, y, z)+\sigma^{M_{y}}(x, y, z)
$$

There, $\sigma^{N}(x, y, z)=\varepsilon^{N}(x) E(x, y, z)=\frac{\sigma^{N}(x)}{E_{L}^{N H}(x)} E(x, y, z)$ is the normal stress caused by normal force and $\sigma^{N}(x)=\frac{N(x)}{A}$ is the effective normal stress in homogenized cross-section while constant effective normal strain $\varepsilon^{N}(x)=\frac{\sigma^{N}(x)}{E_{L}^{N H}(x)}$ over the whole cross-sectional area is assumed. Here $E_{L}^{N H}(x)$ is the effective elasticity modulus for tension-compression [7].

If the effective bending stiffness about the axis $z$ is $D_{z}(x)=I_{z} E_{L}^{M_{z}}(x)$, then the distribution of the effective bending strain is $\varepsilon^{M_{z}}(x, y)=\frac{M_{z}(x)}{D_{z}(x)} y$ and the effective bending stress is $\sigma^{M_{z}}(x, y)=\varepsilon^{M_{z}}(x, y) E_{L}^{M_{z}}(x)$. The real bending stress at position $(x, y, z)$ is 
$\sigma^{M_{z}}(x, y, z)=\varepsilon^{M_{z}}(x, y) E(x, y, z)=\frac{E(x, y, z)}{E_{L}^{M_{z}}(x)} \sigma^{M_{z}}(x, y)$. Here, $E_{L}^{M_{z}}(x)$ is the effective elasticity modulus for bending about z-axis [7].

Analogically, if the effective bending stiffness about the axis $y$ is $D_{y}(x)=I_{y} E_{L}^{M_{y}}(x)$, then the distribution of the effective bending strain is $\varepsilon^{M_{y}}(x, z)=\frac{M_{y}(x)}{D_{y}(x)} z$ and the effective bending stress is $\sigma^{M_{y}}(x, z)=\varepsilon^{M_{y}}(x, z) E_{L}^{M_{y}}(x)$. The real bending stress at position $(x, y, z)$ is $\sigma^{M_{y}}(x, y, z)=\varepsilon^{M_{y}}(x, z) E(x, y, z)=\frac{E(x, y, z)}{E_{L}^{M_{y}}(x)} \sigma^{M_{y}}(x, z)$. Here, $E_{L}^{M_{y}}(x)$ is the effective elasticity modulus for bending about z-axis [7]. It is not simply obtaining functional distribution of the normal stress. For discontinuous variation of the material properties in transversal and lateral direction, the stress has to be calculated at discrete points of crosssection.

\section{Numerical experiments}

In the chapter, the results of numerical investigations concerning the normal stress calculation in the FGM beams with spatially varying material properties are presented.

\subsection{Normal stress calculation in the FGM cantilever beam}

The cantilever FGM beam is considered (as shown in Fig. 4), which is loaded at its free end by forces $F_{y}=F_{z}=10 \mathrm{~N}$ and $F_{x}=-5 \mathrm{kN}$ and by torsion moment $M_{x}=10 \mathrm{Nm}$. Its rectangular cross-section is constant with height $h=0.005 \mathrm{~m}$ and width $b=0.01 \mathrm{~m}$. The length of the beam is $L=0.1 \mathrm{~m}$. The local coordinates system is denoted by the axis $x, y, z$.

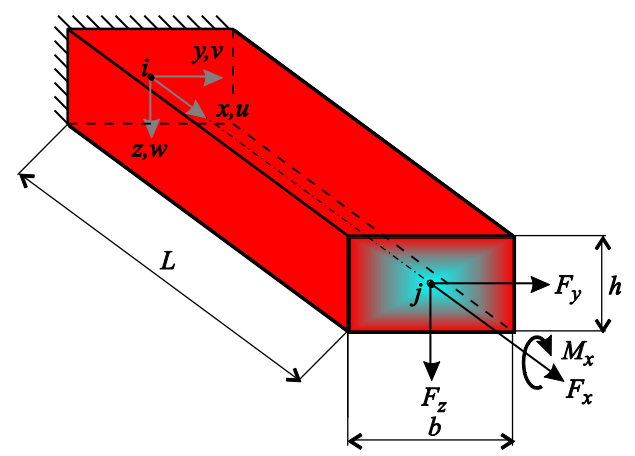

Fig. 4. Cantilever FGM beam with spatial variation of material properties

Material of the beam consists of two components: Aluminum A16061-TO - denoted with index $m$ and Titanium carbide TiC -denoted with index $f$. The material properties of the components are assumed to be constant and their values are: Aluminum Al6061-TO the elasticity modulus $E_{m}=69.0 \mathrm{GPa}$, the Poisson's ratio $v_{m}=0.33$; Titanium carbide TiC - the elasticity modulus $E_{f}=480.0 \mathrm{GPa}$, the Poisson's ratio $v_{f}=0.20$.

The $\mathrm{TiC}$ volume fraction varies in the $y$ and $z$ direction linearly and symmetrically according to the $x-y$ and $x$-z planes: $\left|v_{f}(y, z)\right|_{z=0}^{y=0}=0,\left[\left.v_{f}(y, z)\right|_{\substack{y= \pm h / 2 \\ z= \pm b / 2}}=1-\right.$ the core of the beam 
is made from pure Al6061-TO and linearly varies to the edges that are made from pure TiC. Constant effective material properties are considered in the local $x$-direction of the beam. The average shear correction factors in $\mathrm{y}-$ direction $k_{y}^{s m}=5 / 6$ and in $z$-direction $k_{z}^{s m}=5 / 6$ have been considered (constant Poisson ratio has been assumed in the example).

Using the EMR and MLM, the homogenized elasticity modules (in [GPa]) for: axial loading - $E_{L}^{N H}=342.8 \mathrm{MPa}$, bending about axis $y$ and $z-E_{L}^{M_{y} H}=E_{L}^{M_{z} H}=397.5 \mathrm{MPa}$, shear $-G_{L y}^{H}=E_{L z}^{H}=138.9 \mathrm{MPa}$, and torsion $-G_{L}^{M_{x} H}=162.6 \mathrm{MPa}$ have been calculated [15].

The FGM beam, clamped at the node $i$, has been studied by the elastic-static and buckling analysis. All the calculations were done with our 3D FGM beam finite element (NFE) which we have implemented into the code MATHEMATICA [16]. Additionally, the effect of axial force was considered. It has to be pointed out that the entire structure is discretized using only one our finite element. The critical buckling force is $N_{K i}^{I I}=10.28 \mathrm{kN}$. In the elasto-static analysis the axial force (tension and compression) $N^{I I} \equiv N$ have been chosen as a part of the critical buckling force $N_{K i}^{I I}$. The same problem has been solved using a very fine mesh - 28889 of SOLID186 elements of the FEM program ANSYS [17]. The results of ANSYS as well as the results of the NFE are presented in Table 1. The average relative difference $\Delta[\%]$ between displacements calculated by our method and the ANSYS solution has been evaluated.

Table 1. Displacements and rotations at the free beam end

\begin{tabular}{|c|c|c|c|c|c|c|}
\hline $\begin{array}{c}\text { Displacements } \\
{[\mathbf{m m}],[\mathbf{r a d}]}\end{array}$ & $\begin{array}{c}\mathbf{N F E} \\
\boldsymbol{F}_{\boldsymbol{x}}=\mathbf{0} \mathbf{N}\end{array}$ & $\begin{array}{c}\text { ANSYS } \\
\boldsymbol{F}_{\boldsymbol{x}}=\mathbf{0} \mathbf{N}\end{array}$ & $\boldsymbol{\Delta}[\mathbf{\%}]$ & $\begin{array}{c}\mathbf{N F E} \\
\boldsymbol{F}_{\boldsymbol{x}}=-\mathbf{5 k N}\end{array}$ & $\begin{array}{c}\text { ANSYS } \\
\boldsymbol{F}_{\boldsymbol{x}}=-\mathbf{5 k N}\end{array}$ & $\Delta[\mathbf{\%}]$ \\
\hline$u_{j}$ & 0 & 0 & --- & -0.029230 & -0.029385 & 0.53 \\
\hline$v_{j}$ & 0.020349 & 0.020271 & 0.38 & 0.022281 & 0.022173 & 0.49 \\
\hline$w_{i}$ & 0.080879 & 0.080645 & 0.29 & 0.158012 & 0.157130 & 0.56 \\
\hline$\varphi_{x i}$ & 0.021533 & 0.021844 & 1.42 & 0.021534 & 0.021185 & 1.47 \\
\hline$\varphi_{v i}$ & -0.001211 & -0.001206 & 0.43 & -0.000346 & 0.000344 & 0.75 \\
\hline$\varphi_{z i}$ & 0.000303 & 0.000302 & 0.03 & 0.002417 & 0.002410 & 0.26 \\
\hline
\end{tabular}

As is shown in Table 1, the accuracy of our results is excellent compared to the ANSYS solution. The bending moments about the $y$ and $z$ - axis, the torsional moment, the axial, transversal and shear forces are calculated. To save space, the distribution of the bending moment about $y$-axis is shown in Fig. 5 only. As expected, the axial force $F_{x}$ influence distribution of the bending moment significantly. The high efficiency of our method (NFE) is obvious since our results are evaluated using only one finite beam element compared to the large number of 41924 elements used in the continuum mesh (ANSYS).

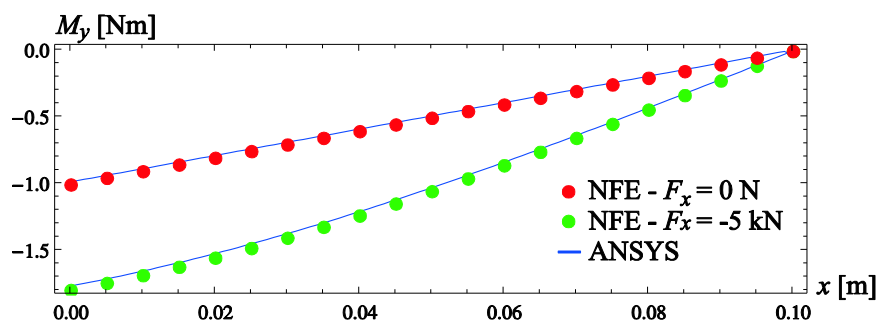

Fig. 5. Bending moment about the $y$ - axis 
Due to stress calculation, the internal forces at the clamped end $(x=0)$ are evaluated and they are: $M_{x}=10 \mathrm{Nm} ; M_{y}=1.78 \mathrm{Nm} ; M_{z}=1.12 ; N_{x}=-5 \mathrm{kN} ; Q_{y}=Q_{z}=10 \mathrm{~N}$.

According the expressions in chapter 2 , the normal stress caused by axial force are calculated. Because of discontinuous variation of elasticity modulus in real beam, the cross section is discretized on layers with constant elasticity modulus - Fig. 6.

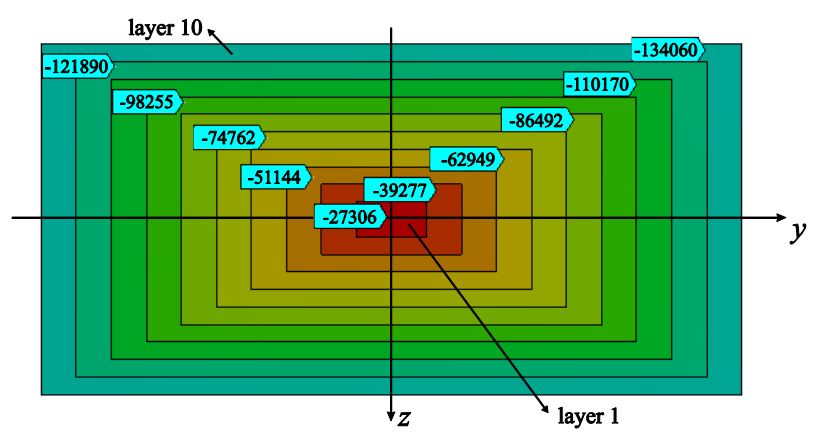

Fig. 6. Compression normal stress distribution caused by the axial force

The maximal axial force stress are in the layer number 10 and minimal in layer 1 - see Table 2. Accuracy of the calculation approach depends of the number of the layers. As shown in Table 2, for then layers is the difference between our results (NFE) and the ones obtained by very fine mesh of 3D-ssolid FE (ANSYS) very small. By very fine division to layers a continuous distribution of the stress can be obtained.

Table 2. Axial force normal stress at the clamped cross-section

\begin{tabular}{|c|c|c|c|}
\hline Layer & $\begin{array}{c}\sigma[\mathbf{k P a}] \\
\mathbf{N F E}\end{array}$ & $\begin{array}{c}\sigma[\mathbf{k P a}] \\
\text { ANSYS }\end{array}$ & $\Delta[\%]$ \\
\hline 1 & -26175 & -27306 & 4.14 \\
\hline 2 & -38189 & -39277 & 2.70 \\
\hline 3 & -50203 & -51144 & 1.84 \\
\hline 4 & -62216 & -62949 & 1.16 \\
\hline 5 & -74230 & -74762 & 0.71 \\
\hline 6 & -86244 & -86492 & 0.29 \\
\hline 7 & -98258 & -98255 & 0.01 \\
\hline 8 & -110272 & -110170 & 0.09 \\
\hline 9 & -122285 & -121890 & 0.32 \\
\hline 10 & -134299 & -134060 & 0.18 \\
\hline
\end{tabular}


Distribution of the bending normal stress is shown in Fig. 7 and Table 3.
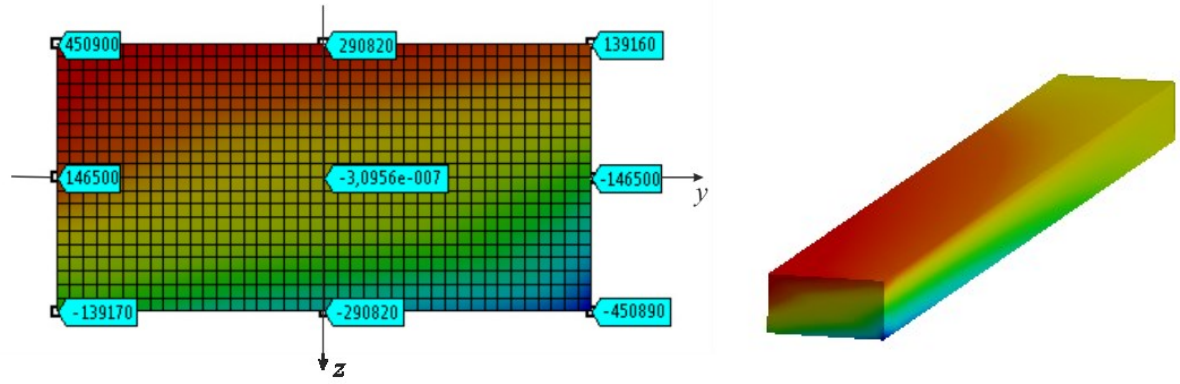

Fig. 7. Resultant biaxial bending normal stress calculated by ANSYS

Table 3. Comparing of the biaxial bending normal stress at chosen position in clamped cross-section

\begin{tabular}{|c|c|c|c|}
\hline Position & $\begin{array}{c}\sigma[\mathbf{k P a}] \\
\mathbf{N F E}\end{array}$ & $\begin{array}{c}\sigma[\mathbf{k P a}] \\
\text { ANSYS }\end{array}$ & $\boldsymbol{\Delta}[\mathbf{\%}]$ \\
\hline$y=0, z=0$ & 0 & 0 & 0 \\
\hline$y=0, z=-h / 2$ & 290594 & 290820 & 0.08 \\
\hline$y=0, z=h / 2$ & -290594 & -290820 & 0.08 \\
\hline$y=-b / 2, z=0$ & 145297 & 146500 & 0.83 \\
\hline$y=b / 2, z=0$ & -145297 & -146500 & 0.83 \\
\hline$y=-b / 2, z=-h / 2$ & 435891 & 450900 & 3.44 \\
\hline$y=b / 2, z=h / 2$ & -435891 & -450900 & 3.44 \\
\hline $\mathrm{y}=b / 2, z=-h / 2$ & 145297 & 139170 & 4.22 \\
\hline$y=-b / 2, z=h / 2$ & -145297 & -139170 & 4.22 \\
\hline
\end{tabular}

\subsection{Normal stress calculation in the FGM cantilever beam on elastic foundation}

The cantilever FGM beam on varying Winkler foundation is considered (as shown in Fig. 8). Its rectangular cross-section is constant with height $h=0.005 \mathrm{~m}$ and width $b=$ $0.01 \mathrm{~m}$. The length of the beam is $L=0.1 \mathrm{~m}$.

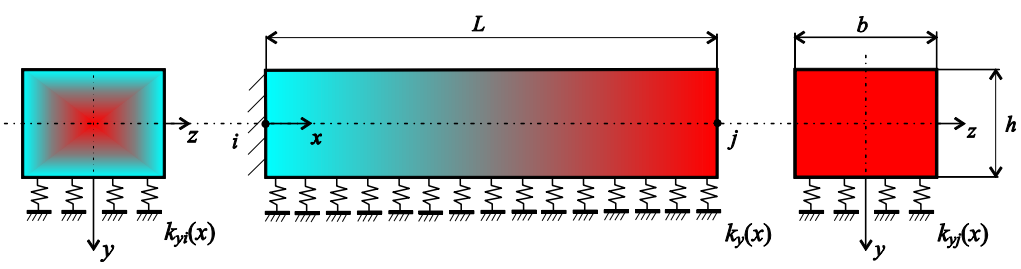

Fig. 8. Cantilever beam on elastic foundation with spatially varying material properties

The beam is made of a mixture of two components: Aluminum Al6061-TO and Titanium Carbide TiC, their constant constituent's material properties are given in Case I. The Aluminum volume fraction, in this case, varies linearly and symmetrically according to 
the $x-y$ and $x-z$ planes: At node $i$ is $\left\langle\left. v_{f i}(y, z)\right|_{\substack{y=0 \\ z=0}}=1,\left\lfloor\left. v_{f i}(y, z)\right|_{\substack{y= \pm h / 2 \\ z= \pm b / 2}}=0\right.\right.$ and then vary continuous linearly in the longitudinal direction to the constant value at node $j\left(v_{f i}=1\right)$.

Using EMR and MLM with $n=20$ layers the effective elastic modulus for axial loading $E_{L}^{N H}$, for bending about axis $y-E_{L}^{M_{y} H}$ and about axis $z-E_{L}^{M_{z} H}$, shear modules $G_{L y}^{H}$ and $G_{L z}^{H}$ and torsional shear modulus $G_{L}^{M_{x} H}$.

$$
\begin{aligned}
& E_{L}^{N H}(x)=342.109-3731.095 x \mathrm{GPa} ; \\
& E_{L}^{M_{y} H}(x)=E_{L}^{M_{z} H}(x)=396.429-3274.293 x \mathrm{GPa} ; \\
& G_{L y}^{H}(x)=G_{L z}^{H}(x)=138.581-1129.418 x \mathrm{GPa} ; \\
& G_{L}^{M_{x} H}(x)=162.233-1362.936 x \mathrm{GPa} .
\end{aligned}
$$

The FGM cantilever beam has been studied by elastic-static analysis. All the calculations were done with our 3D FGM beam finite element (NFE). Additionally, the effect of axial force was considered. It has to be pointed out that the entire structure is discretized using only one herein proposed finite element. The cantilever FGM beam resting on varying vertical Winkler elastic foundation $k_{y}=5000-30000 x+600000 x^{2}$ $\mathrm{kN} / \mathrm{m} 2$ is loaded by forces $F_{y}=F_{z}=50 \mathrm{~N}$ and $F_{x}=-2 \mathrm{kN}$ at node $j$ (Fig. 9). The average shear correction factors in $y^{\prime}$-direction $k_{y}^{s m}=5 / 6$ and in $z^{\prime}$-direction $k_{z}^{s m}=5 / 6$ are used [12]. The displacements at node $\mathrm{j}$ are evaluated using the only one new FGM beam finite element (NFE). The same problem is solved using a very fine mesh -23015 of SOLID186 elements of the FEM program ANSYS [18 34]. The results of ANSYS as well as the results of the NFE are presented in Table 4 . The average relative difference $\Delta[\%]$ between displacements calculated by our method and the ANSYS solution is evaluated.

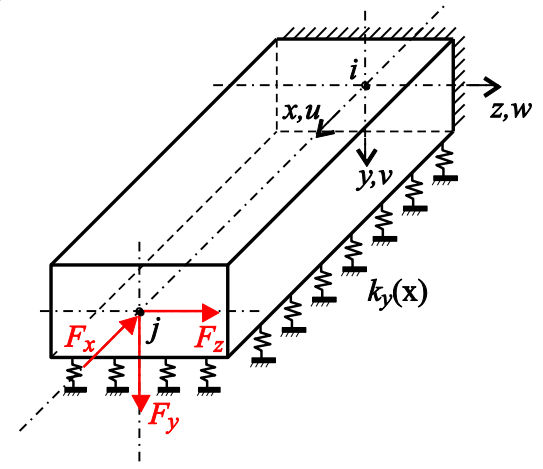

Fig. 9. Loaded FGM beam

Table 4. Displacements at node $j$ with and without elastic foundation

\begin{tabular}{|c|c|c|c|c|}
\hline $\begin{array}{c}\text { Displacement } \\
\text { s at node } \boldsymbol{j} \\
\text { [mm], [rad] }\end{array}$ & $\begin{array}{c}\text { NFE } \\
\text { with } \\
\text { foundation }\end{array}$ & $\begin{array}{c}\text { ANSYS } \\
\text { with } \\
\text { foundation }\end{array}$ & $\begin{array}{c}\text { NFE } \\
\text { without } \\
\text { foundation }\end{array}$ & $\begin{array}{c}\text { ANSYS } \\
\text { without } \\
\text { foundation }\end{array}$ \\
\hline$u_{j}$ & -0.02445 & -0.02507 & -0.02445 & 0.02507 \\
\hline$v_{j}$ & 0.24641 & 0.24933 & 0.74414 & 0.75348 \\
\hline$w_{j}$ & 0.14452 & 0.14791 & 0.14452 & 0.14791 \\
\hline$\varphi_{y j}$ & -0.00247 & -0.00256 & -0.00247 & -0.00256 \\
\hline
\end{tabular}




\begin{tabular}{|c|c|c|c|c|}
\hline $\begin{array}{c}\text { Displacement } \\
\text { s at node } \boldsymbol{j} \\
\text { [mm], [rad] }\end{array}$ & $\begin{array}{c}\text { NFE } \\
\text { with } \\
\text { foundation }\end{array}$ & $\begin{array}{c}\text { ANSYS } \\
\text { with } \\
\text { foundation }\end{array}$ & $\begin{array}{c}\text { NFE } \\
\text { without } \\
\text { foundation }\end{array}$ & $\begin{array}{c}\text { ANSYS } \\
\text { without } \\
\text { foundation }\end{array}$ \\
\hline$u_{j}$ & -0.02445 & -0.02507 & -0.02445 & 0.02507 \\
\hline$v_{j}$ & 0.24641 & 0.24933 & 0.74414 & 0.75348 \\
\hline$w_{j}$ & 0.14452 & 0.14791 & 0.14452 & 0.14791 \\
\hline$\varphi_{z j}$ & 0.00503 & 0.00527 & 0.1304 & 0.01321 \\
\hline
\end{tabular}

The comparison of the vertical beam deflection curve with and without elastic foundation is shown in Fig. 10.

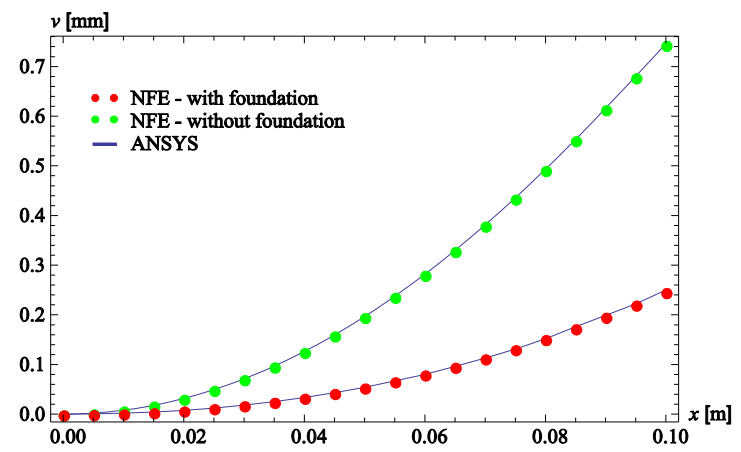

Fig. 10. Vertical beam deflection curve with and without elastic foundation

The bending moments about the $y$ and $z$ - axis for case without elastic foundation are shown in Fig. 11 and Fig. 12, respectively. The Fig. 13 and Fig. 14 show the transversal force in $y$ and $z$-axis. The comparison of the bending moments $M_{y}(x=0), M_{z}(x=0)$ and transversal forces $R_{y}(x=L), R_{y z}(x=L)$ for the case $F_{x}=-2 \mathrm{kN}$ calculated by our approach and by ANSYS are compared in Table 5.

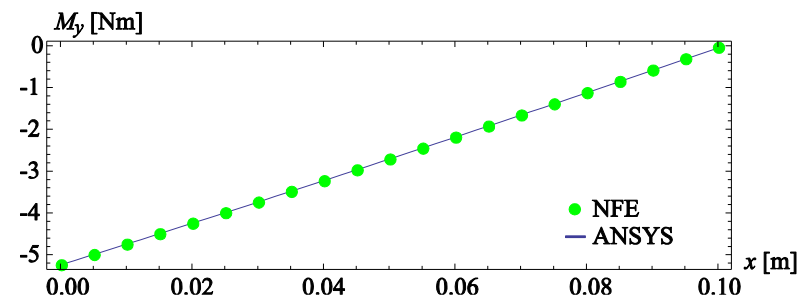

Fig. 11. Bending moment about the $y-$ axis (without elastic foundation)

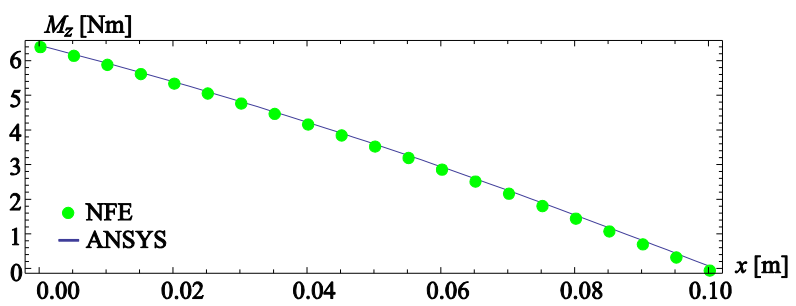

Fig. 12. Bending moment about the $z$-axis (without elastic foundation) 


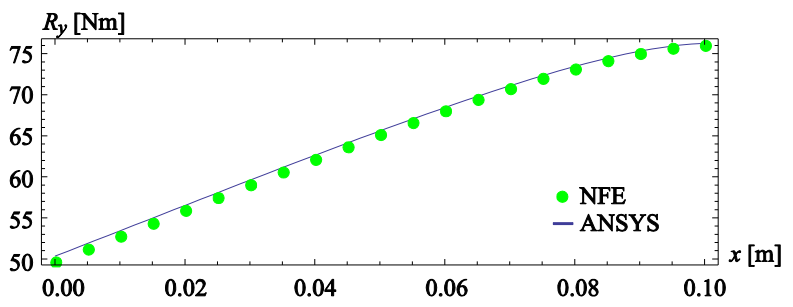

Fig. 13. Transversal force in $y$ - axis (without elastic foundation)

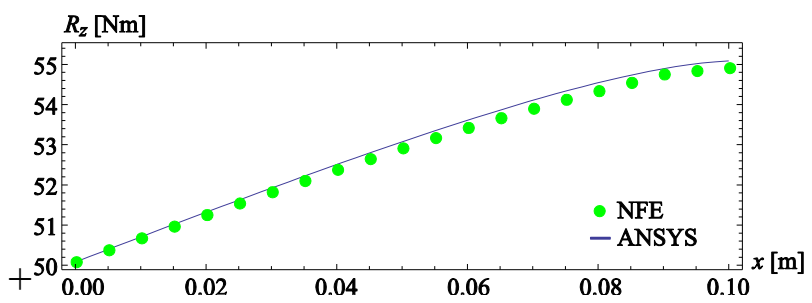

Fig. 14. Transversal force in $z$-axis (without elastic foundation)

Table 5. Bending moments and transversal forces

\begin{tabular}{|c|c|c|c|}
\hline Internal variables & $\begin{array}{c}\mathbf{N F E} \\
\boldsymbol{F}_{\boldsymbol{x}}=\mathbf{- 2} \mathbf{~ k N}\end{array}$ & $\begin{array}{c}\text { ANSYS } \\
\boldsymbol{F} \mathbf{x}=\mathbf{- 2} \mathbf{~ k N}\end{array}$ & $\Delta[\mathbf{\%}]$ \\
\hline$M_{y}(x=0)[\mathrm{Nm}]$ & -5.2182 & -5.2416 & 0.45 \\
\hline$M_{z}(x=0)[\mathrm{Nm}]$ & 6.4691 & 6.4404 & 0.45 \\
\hline$R_{y}(x=L)[\mathrm{N}]$ & 54.9472 & 55.0873 & 0.25 \\
\hline$R_{z}(x=L)[\mathrm{N}]$ & 76.2088 & 76.2251 & 0.02 \\
\hline
\end{tabular}

In Table 6 the comparison of the resultant normal stress, caused by axial, transversal and lateral forces, in the clamped cross-section $(x=0)$ calculated by our approach (Fig. 15) and by ANSYS is shown.

Table 6. Comparison of the normal stress at chosen position in clamped cross-section

\begin{tabular}{|c|c|c|}
\hline position & $\begin{array}{c}\boldsymbol{\sigma}[\mathrm{kPa}] \\
\mathbf{N F E}\end{array}$ & $\begin{array}{c}\boldsymbol{\sigma}[\mathrm{kPa}] \\
\text { ANSYS }\end{array}$ \\
\hline$y=h / 2, z=b / 2$ & -320.40 & -324.21 \\
\hline$y=-h / 2, z=-b / 2$ & 208.20 & 209.35 \\
\hline$y=h / 2, z=b / 2$ & -245.20 & -249.32 \\
\hline$y=0, z=-b / 2$ & 19.80 & 21.56 \\
\hline$y=h / 2, z=0$ & -245.20 & -249.32 \\
\hline$y=-h / 2, z=0$ & 131.50 & 136.22 \\
\hline
\end{tabular}




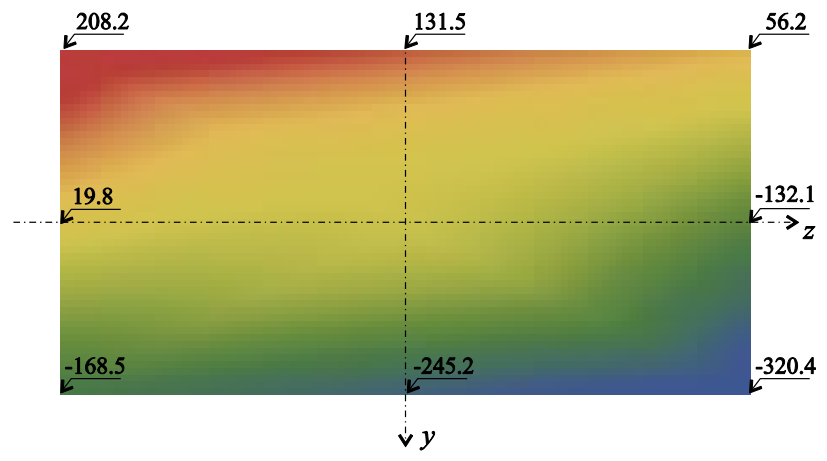

Fig. 15. Resultant normal stress $\sigma(x, y, z)[\mathrm{MPa}]$ at position $x=0$ calculated by our approach

In Fig. 16, the resultant normal stress, caused by axial, transversal and lateral forces (marked in blue flags), in the clamped cross-section is shown that was calculated by ANSYS (using a very fine mesh - 23015 of SOLID186). As can be seen, a very good agreement of both solution method has been obtained at all marked points excluding the corners. As is well known, the solutions with 3D solid finite elements produce in the sharp corners incorrect stress first of all by the very fine meshes. In the nearby points of the sharp corners is the match of the results very good (see the details in Fig. 16).

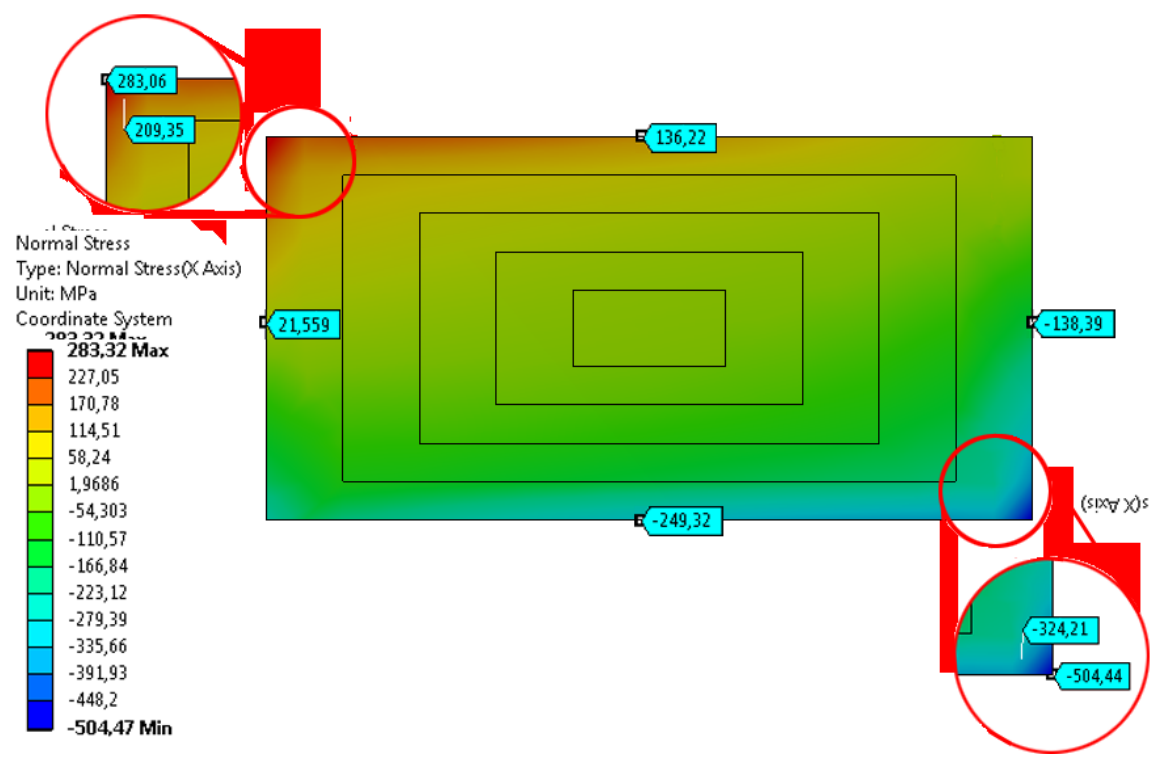

Fig. 16. Normal stress $\sigma(x, y, z)[\mathrm{MPa}]$ at position $x=0$ calculated by ANSYS (SOLID186)

\section{Conclusions}

In this contribution, our new 3D beam finite element for elasto-static and buckling analysis of the FGM single beam and beam structures has been used for calculation of the deformation and internal forces and moments in FGM beams with spatial variation of material properties. Effect of varying Winkler elastic foundations and shear force deformation effect is taken into account. The effect of axial force has been taken into account for the flexural loading. For an elastic-static analysis, the expressions for 
distribution of normal stress on the beam cross-section is established and applied. The obtained results have been studied and compared with results obtained using very fine continuum and beam meshes by the FEM program ANSYS. An excellent agreement of our solution results is obtained, which confirms respectable accuracy and effectiveness of our approach.

This work was financially supported by grants of Scientific Grant Agency of the Ministry of Education of Slovak Republic and the Slovak Academy of Sciences and VEGA No. 1/0453/15.

\section{References}

1. V. Kahya, M. Turan, Finite element model for vibration and buckling of functionally graded beams based on the first-order shear deformation theory. Composites Part B 109, 108-115 (2017)

2. N. Tan-Tien, T.T. Pham, J. Lee, Lateral buckling analysis of thin walled functionally graded open-section beams. Compos. Struct. 160, 952-963 (2017)

3. M. Simsek, Bi-directional functionally graded materials (BDFGMs) for free and forced vibration of Timoshenko beams with various boundary conditions. Compos. Struct. 133, 968-978 (2015)

4. T. Kocaturk, M. Simsek, S.D. Akbas, Large displacement static analysis of a cantilever Timoshenko beam composed of functionally graded material. Sci Eng Compos Mater 18, 21-34 (2011)

5. M. Meradjah, A. Kaci, M.S.A. Houari, A. Tounsi, S.R. Mahmoud, A new higher order shear and normal theory of fuctionaly graded beams. Steel Compos Struct 18, 793-809 (2015)

6. V. Kutis, J. Murin, R. Belak, J. Paulech, Beam element with spatial variation of material properties for multiphysics analysis of functionally graded materials. Comput. Struct. 89, $1192-1205$ (2011)

7. J. Murin, V. Kutis, Improved mixture rules for composite (FGMs) sandwich beam finite element. In Computational Plasticity IX. Fundamentals and Applications. Barcelona, Spain, 647-650 (2007)

8. A.E. Alshorbagy, M.A. Eltaher, F.F. Mahmoud, Free vibration of a functionally graded beam by finite element method. Appl. Math. Modell. 35, 412 - 425 (2010)

9. M. Simsek, Vibration analysis of a functionally graded beam under a moving mass by using different beam theories. Compos. Struct. 92, 904-917 (2010)

10. T. Rout, On the dynamic stability of functionally graded material under parametric excitation. PhD thesis. National Institute of Technology Rourkela, India (2012)

11. J. Murin, S. Kugler, M. Aminbaghai, J. Hrabovsky, V. Kutis, J. Paulech, Homogenization of material properties of the FGM beam and shells finite elements. In. $11^{\text {th }}$ World Congress on Computational mechanics (WCCM XI), Barcelona, Spain (2014)

12. J. Murin, M. Aminbaghai, J. Hrabovsky, V. Kutis, S. Kugler, Modal analysis of the FGM beams with effect of the shear correction function. Composites Part B 45, 15751582 (2013)

13. J. Murin, M. Aminbaghai, J. Hrabovsky, V. Kutis, J. Paulech, S. Kugler, A new FGM beam finite element for modal analysis. In. $11^{\text {th }}$ World Congress on Computational mechanics (WCCM XI), Barcelona, Spain (2014) 
14. J. Murin, S. Kugler, M. Aminbaghai, J. Hrabovsky, V. Kutis, J. Paulech, Homogenization of material properties of the FGM beam and shell finite elements. In. $11^{\text {th }}$ World Congress on Computational mechanics (WCCM XI), Barcelona, Spain (2014)

15. J. Murin, M. Aminbaghai, J. Hrabovsky, V. Kutis, J. Paulech, $3 D$ beam finite element for elastic-static analysis of the FGM structures. In 8th International Congress of Croatian Society of Mechanics (8th ICCSM), Opatija, Croatia (2015)

16. S. Wolfram MATHEMATICA 5, Wolfram research, Inc., 2003

17. ANSYS Swanson Analysis System, Inc., 201 Johnson Road, Houston, PA 15342/1300, USA 